\title{
Anti-HMGB1 mAb therapy for intracerebral hemorrhage-induced brain injury in rats
}

\author{
Dengli Wang ${ }^{1}$, Keyue Liu ${ }^{1}$, Hidenori Wake ${ }^{1}$, Kiyoshi Teshigawara ${ }^{1}$, Shuji Mori ${ }^{2}$, Masahiro Nishibori ${ }^{1}$ \\ ${ }^{I}$ Department of Pharmacology, Okayama University Graduate School of Medicine, Dentistry and Pharmaceutical \\ Science, Japan, ${ }^{2}$ School of Pharmacy, Shujitsu University, Japan
}

\section{Background}

As one of the most lethal stroke subtypes, intracerebral hemorrhage ( $\mathrm{ICH}$ ) is acknowledged as a serious clinical problem lacking effective treatment. Available evidence from preclinical and clinical studies suggests that inflammatory mechanisms are involved in the progression of ICH-induced secondary brain injury. High mobility group box-1 (HMGB1) is a ubiquitous and abundant nonhistone DNA-binding protein, and is also an important proinflammatory molecule once released into the extracellular space from the nuclei. In the present study, we examined whether anti-HMGB1 mAb can provide neuroprotective effects in a rat model of ICH.

Method

Male Wistar rats were randomly divided into an anti-HMGB1mAb group, a class-matched control mAb group and a sham group. Intracerebral hemorrhage was induced by the local injection of collagenase IV in the striatum of the rats. At the indicated times, blood was taken for ELISA test, d-ROMs and BAP test. Brains were embedded in paraffin for immunofluorescence analysis or were homogenized for western blotting and real time PCR. For brain edema analysis, ipsilateral brains were harvested to detect brain water content. To evaluate BBB permeability, a measurement of Evans blue extravasation was used. Finally, the performance scores at different time points were determined by a grip strength test and a cylinder test.

Result

We show that treatment with neutralizing anti-HMGB1 mAb remarkably ameliorated ICH-injury induced by local injection of collagenase IV in the striatum of rats. Administration of anti-HMGB1 mAb inhibited the release of HMGB1 into the extracellular space in the peri-hematomal region, reduced serum HMGB1 levels and decreased brain edema by protecting blood-brain barrier integrity, in association with decreased activated microglia and the expression of inflammation-related factors at $24 \mathrm{~h}$ after ICH. Consequently, anti-HMGB1 mAb reduced the oxidative stress and improved the behavioral performance of rats. These results strongly indicate that HMGB1 plays a critical role in the development of ICH-induced secondary injury through the amplification of plural inflammatory responses.

Conclusion

Intravenous injection of neutralizing anti-HMGB1 mAb has potential as a novel therapeutic strategy for ICH, even if the treatment is initiated at $3 \mathrm{~h}$ after the onset of hemorrhage. 